\title{
FREE SUBSEMIGROUPS OF DOMAINS
}

\author{
A. A. KLEIN \\ (Communicated by Maurice Auslander)
}

\begin{abstract}
It is proved that the multiplicative semigroup of the ring of polynomials in two commuting indeterminates over a noncommutative domain contains a noncommutative free subsemigroup.
\end{abstract}

The question whether the multiplicative group of a division ring $D$ contains a free noncommutative subgroup has been raised by Lichtman [4]. The answer is positive when $D$ is finite-dimensional over its center $Z$. A more general result is given in [2]. The question is studied further in [3], but the general case is still open. Makar-Limanov raised a seemingly simpler question: Does $D^{*}=D-\{0\}$ contain a free noncommutative subsemigroup? In [5] he proved that the answer is positive when $Z$ is uncountable.

In the present note we propose a more general question: Given a noncommutative domain $D$ (with 1 ) does $D^{*}$ contain a free noncommutative subsemigroup? Using a recent result [1, Theorem 3] it is possible to extend MakarLimanov's result to noncommutative domains. We prove a stronger result that has the advantage that it does not make any assumptions on the center. It says that if $R$ is a noncommutative domain and $u, v$ are commuting indeterminates over $R$, then the multiplicative semigroup of the ring of polynomials $R[u, v]$ contains a free noncommutative subsemigroup. The case of a domain with uncountable center then becomes an easy corollary.

Let $W$ be the free semigroup of words in two letters $x, y$. A nonempty word can be written in the form $x^{i_{1}} y^{j_{1}} \ldots x^{i_{r}} y^{j_{r}}$ with $r, j_{1}, \ldots, i_{r} \geq 1$ and $i_{1}, j_{r} \geq 0$. A relation between two elements $a, b$ of a semigroup is a pair $\left(w_{1}, w_{2}\right)$ of two distinct words $w_{1}, w_{2} \in W$. Since we are interested in the semigroup $S^{*}$ where $S$ is a domain, we may consider only relations $\left(w_{1}, w_{2}\right)$ such that $w_{1}, w_{2}$ do not end (and do not start) with the same letter. A relation is said to be homogeneous if $w_{1}$ and $w_{2}$ have the same degree in $x$ and the same degree in $y$.

In what follows, $R$ will be a domain and $R[u, v]$ the polynomial ring in two commuting indeterminates $u, v$ over $R$. The subring of $R$ generated by 1 is denoted by $P$, and $P\langle x, y\rangle$ is the free algebra in $x, y$ over $P$.

Received by the editors October 12, 1990 and, in revised form, March 25, 1991.

1991 Mathematics Subject Classification. Primary 16U10; Secondary 20M05. 
Lemma. Given $a, b \in R$, if $\left(w_{1}, w_{2}\right)$ is a relation between $a+u$ and $b+$ $v(a+u)$, considered as elements of $R[u, v]$, then this relation is homogeneous and $\left(a d_{a}\right)^{n} b=0$ where $n+1$ is the total degree of the relation.

The proof we give is similar to that of Lemma 2 in [5]. There is some flow in that proof. The assumption made in the statement (the relation holds for $x+c, y+d x)$ is not the one used in the proof (the relation holds for $x+c$, $y+d(x+c))$. For this reason, we repeat some of the arguments and we even get a simpler proof due to our reduction to relations with distinct last letters.

Proof. The equality $w_{1}(a+u, b+v(a+u))=w_{2}(a+u, b+v(a+u))$ yields that the two sides have the same $v$-degree and the same $u$-degree, and this implies that $\left(w_{1}, w_{2}\right)$ is homogeneous. We may assume by symmetry that $w_{1}$ ends with $y$ and $w_{2}$ ends with $x$. If the $y$-degree of the relation is $j$ then we have

$$
w_{1}(x, y+v x)-w_{2}(x, y+v x)=\sum_{q=1}^{j} f_{q}(x, y) v^{j-q}
$$

where $f_{q}(x, y)$ belongs to $P\langle x, y\rangle$ and is homogeneous in $x$ and $y$, and of degree $q$ in $y$. Moreover $f_{q}(a+u, b)=0$ and, in particular, $f_{1}(a+u, b)=0$. We have

$$
f_{1}(x, y)=\sum_{m=0}^{n} \alpha_{m} x^{m} y x^{n-m}
$$

where $n+1$ is the total degree of the relation and $\alpha_{n}=1$ since $w_{1}$ ends with $y$.

Now we proceed to prove by induction on $n$ that if for two elements $a$, $b \in R$ there exists $f_{1}(x, y) \in P\langle x, y\rangle$ as in (2) with $\alpha_{n}=1$ such that $f_{1}(a+u, b)=0$, then $\left(a d_{a}\right)^{n} b=0$. We may assume that $b \neq 0$.

If $n=1$ then $\alpha_{0} b(a+u)+(a+u) b=0$ so $\left(\alpha_{0}+1\right) b u=0$ and $\alpha_{0}=-1$ since $b \neq 0$. Thus we have $\left(a d_{a}\right) b=a b-b a=0$. To proceed from $n-1$ to $n$, we show that there exists $g(x, y)=\sum_{m=0}^{n-1} \beta_{m} x^{m} y x^{n-1-m} \in P\langle x, y\rangle$ with $\beta_{n-1}=1$ such that $g\left(a+u, b_{1}\right)=0$ where $b_{1}=\left(a d_{a}\right) b$. By induction, this will imply $\left(a d_{a}\right)^{n-1} b_{1}=0$ and therefore $\left(a d_{a}\right)^{n} b=0$.

To get $g(x, y)$, let $y_{1}=[x, y]$ so $x y=y x+y_{1}$. Using this relation we transform the left-hand side of (2) into

$$
\sum_{m=0}^{n-1} \beta_{m} x^{m} y_{1} x^{n-1-m}+\beta y x^{n} .
$$

Since $\alpha_{n}=1$, we get $\beta_{n-1}=1$. Let $g(x, y)=\sum_{m=0}^{n-1} \beta_{m} x^{m} y x^{n-1-m}$, then, since $f_{1}(a+u, b)=0$, we have $g\left(a+u, b_{1}\right)+\beta b(a+u)^{n}=0$ where $b_{1}=$ $[a, b]$. But the $u$-degree of $g\left(a+u, b_{1}\right)$ is at most $n-1$, so $\beta=0$ and $g\left(a+u, b_{1}\right)=0$. This completes the proof of the lemma.

Theorem. Let $R$ be a noncommutative domain and $R[u, v]$ the ring of polynomials in two commuting indeterminates over $R$. Then the multiplicative semigroup of $R[u, v]$ contains a free noncommutative subsemigroup.

Proof. If the result is false, then given $a, b \in R$ the elements $a+u, b+$ $v(a+u)$ of $R[u, v]$ satisfy a relation. It follows by the lemma that $\left(a d_{a}\right)^{n} b=0$. 
But by [1, Theorem 3] a domain $R$ with the last property is commutative, a contradiction.

Corollary. Let $S$ be a noncommutative domain with uncountable center $Z$. Then $S^{*}$ contains a free noncommutative subsemigroup.

Proof. Applying the theorem, it suffices to prove that $S$ contains a subring isomorphic to a ring of polynomials $R[u, v]$ where $R$ is a noncommutative subring of $S$.

Let $a, b \in S$ be such that $a b \neq b a$, and let $R$ be the subring generated by $a, b$, and 1 . Thus $R$ is a countable domain. Since a nonzero polynomial of degree $n$ in $R[u]$ has at most $n$ roots in $Z$, we get that the set of roots belonging to $Z$, of all the nonzero polynomials in $R[u]$ is countable. Since $Z$ is uncountable, there exists an element $u_{1} \in Z$ that is transcendental over $R$, so $R\left[u_{1}\right] \simeq R[u]$. Starting with the domain $R_{1}=R\left[u_{1}\right]$, which is also countable, we get by the same argument, that there exists an element $v_{1} \in Z$ transcendental over $R_{1}$. So $R_{1}\left[v_{1}\right] \simeq R[u][v]$ and, therefore, $R\left[u_{1}, v_{1}\right] \simeq R[u, v]$. Thus $R\left[u_{1}, v_{1}\right]$ is the required subring of $S$.

We conclude with several remarks.

1. We have not assumed that the center is a field, but our question may be reduced to this case. Indeed, by localizing at the center we get a domain with the center a field and if the answer is positive for the localized domain then it is positive for the given domain. This follows since one can show that if two elements in $D$ satisfy a relation then they also satisfy a homogeneous relation.

2. Our domain may be assumed to be right and left Ore for if this is not the case, it contains not only a noncommutative free subsemigroup but also a free algebra in two generators. Moreover, we may assume that all the subrings of our domain are Ore.

3. In view of our result, perhaps a first attempt to solve the question would be to consider the polynomial ring in one indeterminate over a noncommutative domain.

\section{REFERENCES}

1. H. E. Bell and A. A. Klein, On the rings with Engel condition, Canad. Math. Bull. 34 (1991), 295-300.

2. J. Z. Gonçalves, Free groups in subnormal subgroups ..., Canad. Math. Bull. 27 (1984), 365-370.

3. J. Z. Gonçalves and A. Mandel, Are there free groups in division rings?, Israel J. Math. 53 (1986), 69-80.

4. A. I. Lichtman, On subgroups of the multiplicative group of skew fields, Proc. Amer. Math. Soc. 63 (1977), 15-16.

5. L. Makar-Limanov, On free subsemigroups of skew fields, Proc. Amer. Math. Soc. 91 (1984), 189-191.

Raymond and Beverly Sackler faculty of Exact Sciences, School of Mathematical Sciences, Tel-Aviv University, Tel Aviv 69978, IsRael

E-mail address: aaklein@math.tau.ac.il 